\title{
Efficient Signal Detection Techniques in TV White Space using Cognitive Radio
}

\author{
Chitra Sapkal $^{1}$, S. D. Borde ${ }^{2}$ \\ ${ }^{1}$ E\&TC Department, P.E.S's Modern College of Engineering, Savitribai Phule Pune University, J.M. Road, Shivajinagar, Pune-411005, \\ India
}

\begin{abstract}
TV white space is the unused TV spectrum which can be used by secondary users to solve the problem of spectrum scarcity and to increase its efficiency. All DTT signals use OFDM, but they many employ different sets of pilot carriers, guard intervals and symbol sizes. The proposed algorithms aim to detect CR signal with reasonable detection performance in TV white space. First algorithm is based on cyclo-stationary feature detection and second algorithm is based on energy detection.
\end{abstract}

Keywords: channel aggregation, cognitive radio, spectrum sensing, TV white space, OFDM.

\section{Introduction}

Cognitive radio (CR) technology has involved particular attention as a noticeable solution for reduction of spectrum resources [1]. It acquires knowledge of surrounding radio environment and regulates its operational parameters and protocols dynamically, thus it could attain efficient utilization of spectrum resources. This is why FCC (Federal Communications Commission) of U.S. and OFCOM of U.K. allowed implementation of CR system in TV white space [2][4].

Since TV white space offers greater radio propagation property compare with the straight ISM (Industrial, Scientific and Medical) band, plenty of industries have communicated their interests on TV white space. Those research interests have leaded various standard events of wireless communication systems in TV white space. IEEE 802, ISOIIEC, and ECMA international are primary standard groups in TV white space.

ECMA-392 is the first standard group which distributed MAC (Medium Access Control) and PHY (Physical layer) specification in TV white space for personnel/portable use [5]. ISO/IEC 16504 is a adapted version of ECMA-392 standard for the same application with comparable system structure. IEEE 802 standard group has some working group (WG) for TV white space [6]. IEEE 802.22 WG has issued a standard for wireless regional area networks (WRAN) [7]. It is a static service to offer internet access in rural area. IEEE 802.11 WG made a task group (TG) to launch an amendment for procedure of WLAN (Wireless Local Area Networks) system in TV white space [8]. The name of TG is IEEE 802.11 af, and it objects to deliver Wi-Fi services in TV white space, which is so called Super Wi-Fi.

Several CR system standards stated above will be set up in TV white space, therefore certain spectrum protocols are needed for coexistence in TV band. IEEE 802.19.1 TG has worked on the problem and prepared a draft document for TV white space coexistence techniques [9]. Criterion of coexistence is acknowledgement of CR system for dynamic strategy implementation. Hence, spectrum sensing ability to detect not only primary services but also CR system is essential for vertical and horizontal coexistence in TV white space.

Up to now, since most of CR systems have hired OFDM (Orthogonal Frequency Division Multiplexing) technologies, CR system sensing schemes are usually based on OFDM signal detection algorithms [10]-[13]. Meanwhile, for finding and organization of CR systems in TV white space, spectrum sensing algorithms using cyclo-stationary feature were suggested [14]. The algorithm can unfailingly sense and classify CR systems in TV white space.

However, the conventional algorithms have a main disadvantage that they cannot identify multi-channel $\mathrm{CR}$ signal. As demands for high-speed wireless communication service growth, most of systems consider multi-channel operation to increase their system throughput. Multi-channel operation means combined channel usage of contiguous or non-contiguous ways. Signal characteristic of multi-channel signal is dissimilar from the single channel signal while the conventional algorithms have no competence to sense the multi-channel signal. For that reason, multi-channel CR signal detection algorithms are basically essential for coexistence of CR systems in TV white space.

In this paper, we goal to implement multi-channel CR system detecting algorithms. The suggested schemes use cyclostationary feature and spectrum shape to sense multi-channel CR signal. With above features, they will detect number of aggregated channels. In addition, they will bring out multichannel signal recognition with the conventional single channel RF (Radio Frequency) front-end. As a result, we can recognize multiple channel status by implementing the proposed algorithms once only for a single channel.

\section{Multichannel Operation of CR system}

Maximum of CR system standards in TV white space consider multi-channel operation to provide better system throughput. Multi-channel process was already considered in the conventional WLAN standard such as IEEE 802.11n. There are two types of multi-channel operation: contiguous 


\section{International Journal of Science and Research (IJSR) \\ ISSN (Online): 2319-7064}

Index Copernicus Value (2013): 6.14 | Impact Factor (2014): 5.611

and noncontiguous channel aggregation. Contiguous channel aggregation uses joint multiple adjacent channels while noncontiguous method uses multiple separated channels simultaneously [3].

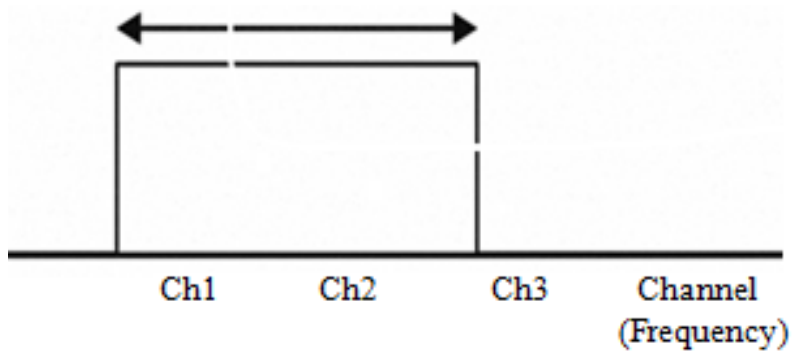

(A) Contiguous channel aggregation

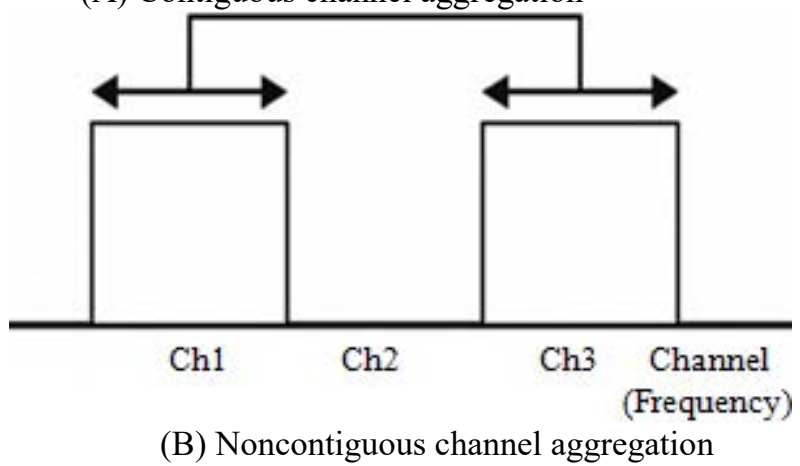

Figure 1: Description of channel aggregation types

Conceptual description of each channel aggregation process is drawn in Fig. 1 The noncontiguous channel aggregation, as described in (B) of Fig.1, combines two disconnected channels logically. In other words, each of noncontiguous channel aggregated signal is identical as the single channel operation signal except that both channels are used simultaneously by one communication link. Therefore, it sustains the same signal characteristic and could be detected with the conventional CR system sensing algorithm.

On the other hand, as described in (A) of Fig.1, contiguous channel aggregation uses multiple sequential channels. As a result, signal characteristic of contiguously aggregated channel may be different from the conventional single channel operation signal, thus new signal detection algorithms for contiguous channel aggregation systems are required. Contiguous channel aggregation is typically called channel bonding.

In addition, for implementation aspect, it is strongly recommended to perform spectrum sensing of channel bonded signal with the conventional single channel detecting receiver for compatibility. Furthermore, computational complexity could be considerably reduced if we could recognize channel bonding information with only single channel signal sensing. In other words, for detection of channel bonded CR system signal which range over multiple channels, if identification of channel bonding information is possible with only one channel sensing, efficient sensing algorithm could be implemented by avoiding the sensing procedure for the rest of channels [3]

\section{Cyclostationary based Channel Bonding Signal Sensing Algorithm}

For detection of channel bonding signal, detection method utilizing cyclo-stationary feature is used. OFDM signal with $\mathrm{Nu}-\mathrm{FFT}$, Nc used sub-carrier, and $\mathrm{Ng}$ guard sub-carrier is defined in digital domain as

$$
s[n]=\sum_{u=-\infty}^{m} g\left(n-u_{s}\right)\left(\sum_{i=0}^{N_{e}-1} d[u, i] e^{2 \pi \frac{i-\left(N_{e}-1\right) / 2}{N_{u}}} n i\right)
$$

where $\mathrm{Ns}(=\mathrm{Nu}+\mathrm{Ng})$ is total time domain samples of a OFDM symbol including guard interval and $d[u, i]$ represents data of $i$-th sub-carrier in $u$-th OFDM symbol. In equation (1), $\mathrm{g}[\mathrm{n}]$ is defined as below.

$$
\mathrm{g}[\mathrm{n}]=\left\{\begin{array}{l}
1,0 \leq \mathrm{n}<\mathrm{N}_{s} \\
0, \text { otherwise }
\end{array}\right\}
$$

As refer to [16], cyclo-stationary feature of equation (1) could be represented with cyclic frequency $\alpha_{k}$ and time interval $^{1}$ as

$$
\mathrm{R}_{s s}^{\alpha_{\mathrm{k}}}[1]=\sigma_{\mathrm{d}}^{2} \mathrm{~A}[\mathrm{l}]{\frac{\sin \left\lfloor\pi \alpha_{\mathrm{k}}\left(\mathrm{N}_{s}-|1|\right)\right\rfloor}{\pi \mathrm{k}}}_{\text {0, otherwise }} \text { for }|1| \leq \mathrm{N}_{s}
$$

Where $\sigma^{2}$ is expectation of $\left\{d[n, i] . d^{*}[n, i]\right\}$, and

$$
A[1]=\sum_{i=0}^{N_{c^{-1}}} e^{-j 2 \pi\left[\frac{\mathrm{i}-\left(\mathbb{N}_{c}-1\right) / 2}{\mathbb{N}_{u}}\right]} 1
$$

From the equation (3), each channel bonding system represents different cyclo-stationary features according to the number of bonded channels [3], [8].

\section{Energy Detection based Channel Bonding Signal Sensing Algorithm}

Channel bonding system increases size of FFT according to the number of bonded channels. Accordingly, we propose another spectrum sensing algorithm for channel bonding signal.

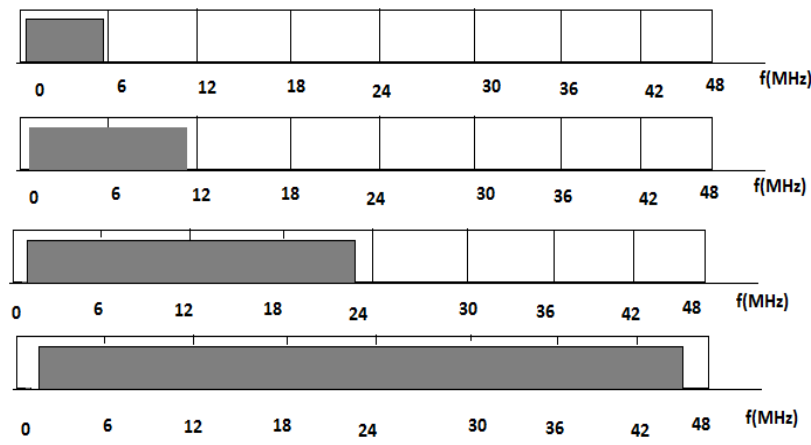

Figure 2: Spectrum shape of channel bonded signal

Fig. 2 represents bands of channel bonding signal define signal spectrum of single channel, two channel bonded, four channel bonded and eight channel bonded signal, respectively. From the figure, we can realize that guard band 


\section{International Journal of Science and Research (IJSR) \\ ISSN (Online): 2319-7064}

Index Copernicus Value (2013): 6.14 | Impact Factor (2014): 5.611

of signal increases according to the actual signal bandwidth. In other words, we can sense and categorize channel bonding signal using the size differences of guard bands.

In aspect of spectrum management, we can normally assume that spectrum sensing process will be carried out for the entire TV channels with the ascending order of channel numbers. With the hypothesis, CR system with single channel sensing RF receiver will receive the left edge $6 \mathrm{MHz}$ spectrum. As a result, signal spectrum shape received by the single channel sensing RF receiver will be different for number of bonded channels as described in Fig. 2. Therefore, we can detect and identify the channel bonding signal by valuing the signal guard bandwidth.

To estimate guard band of the signal, received leftmost channel signal energy of bonded channel signal is used for decision of guard bandwidth [3][9]. Energy detection can be performed with the following formula [2]

$$
V=\sum_{n=1}^{N-1}|R[n]|^{2}>\gamma
$$

Where, $\mathrm{R}[\mathrm{n}]$ is the received signal, $\gamma$ is the predefined threshold and $\mathrm{V}$ is the detected energy.

The number of bonded channels will be decided with above criteria. With grouping of two proposed schemes, we can sense and categorize all existing CR system signal including multi-channel signal.

\section{Performance Analysis}

There are two proposed channel bonding signal sensing algorithms. Out of those first algorithm is implemented and its results are shown in Fig. 4 and Fig. 5. Test vector signal for simulation is acknowledged by single channel RF receiver.

For performance evaluation of channel bonding detection method, OFDM signal is used for test vector. The detection threshold is set to satisfy the false alarm probability of $10 \%$. Since there is no conventional method to detect channel bonded signal, performance verification is accomplished by comparing detection probability of suggested algorithm for different number of bonded channels.

Here, random data is generated and it is converted into OFDM signal with guard band data. It is passed through a normal channel with white Gaussian noise added in it. Received signal is demodulated and autocorrelation of received guard data is calculated to find out cyclostationary feature. So spectral correlation function can differentiate noise energy from primary signal energy. Fig. 3 shows OFDM modulated signal for $\mathrm{N}=1024$ and $\mathrm{GI}=256$ where $\mathrm{N}$ represents data size and GI represents guard interval. On original data PSK modulation is implemented. As the channel bandwidth increases, guard bandwidth increases.

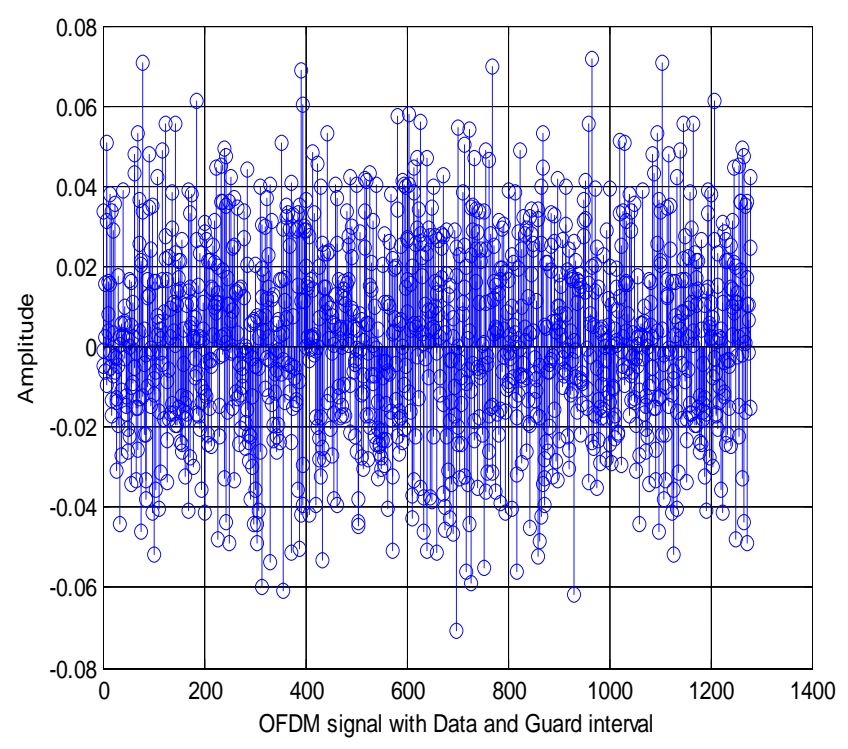

Figure 3: OFDM modulated signal with 1024 data samples and 256 guard interval samples.

In Fig. 4 and fig. 5, detection performance of cyclostationary based channel bonding signal sensing algorithm is verified with simulation results. The $\mathrm{x}$-axis of the figure defines received SNR of input signal for simulation and the $y$-axis represents detection probability of simulation.

First we have find out the detection probabilities of single channel. Fig.4 shows detection performance for five single channels with different values of threshold. So here TC represents threshold of cyclostationary detector and its value increases from $\mathrm{TC} 1$ to TC5. As the threshold increases, probability of false alarm decreases and hence probability of detection of white space increases.

For performance comparison, single channel, two channel bonded, four channel bonded, and eight channel bonded signals are employed.

Detection performance of one, two, four and eight channel bonded signal is plotted simultaneously in fig.5. Here solid lines are used to represent cyclostationary based CBSS algorithm. From Fig. 5, we can confirm that the proposed algorithm identifies channel bonding information without loss of detection. This is possible because each different channel bonded signal represents unique cyclo-stationary feature. On the other hands, since the algorithm only can get partial channel signal information for multiple channel bonded signal, the detection performance of the algorithm degraded as the number of bonded channels increases. 


\section{International Journal of Science and Research (IJSR) \\ ISSN (Online): 2319-7064}

Index Copernicus Value (2013): 6.14 | Impact Factor (2014): 5.611

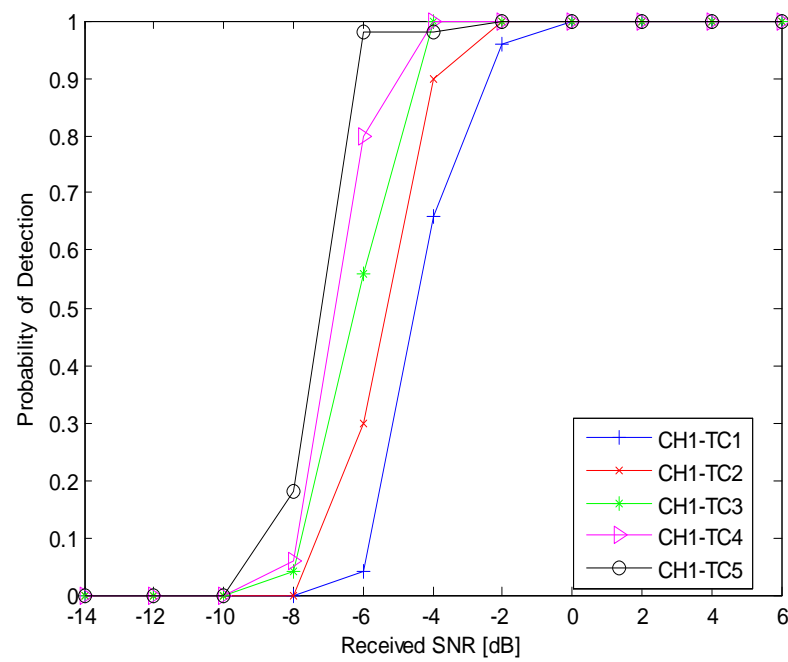

Figure 4: Detection performance of Cyclostationary based channel bonding for single channel with different values of Threshold.

However, the performance loss is negligible especially as number of bonded channel increases, and the detection performance is still reliable even though the performance losses

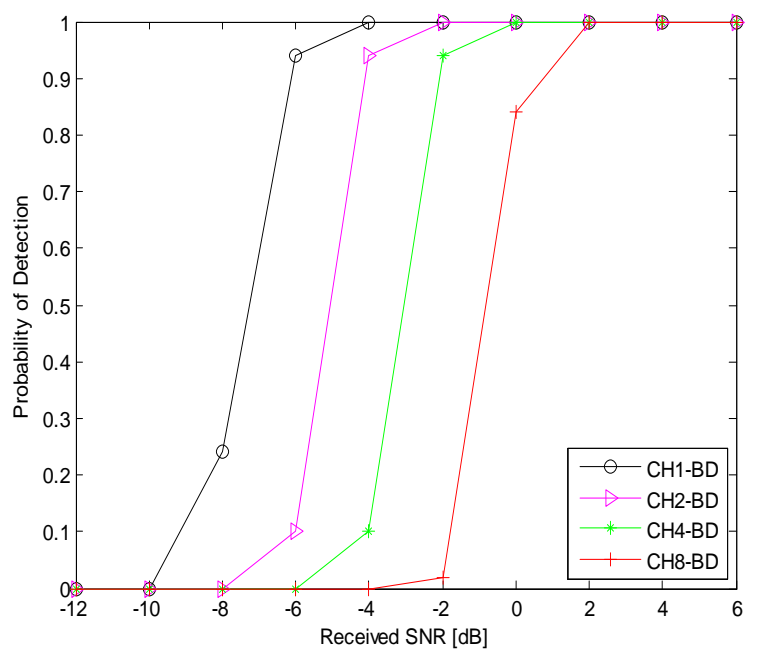

Figure 5: Detection performance for channel bonding using Cyclostationary based CBSS algorithm (For TC5).

Detection of channel bonding information can be identified from the cyclo-stationary feature. On the other hands, using the estimated spectrum shape using Energy detection based signal sensing algorithm, identification of channel bonding is possible.

\section{Conclusion}

Here, efficient multi-channel spectrum sensing algorithm is implemented for cognitive radio systems in TV white space. Utilizing the cyclo-stationary feature algorithm can detect channel bonded signal and identify channel bonding information simultaneously. Since the algorithm only can get partial channel signal information for multiple channel bonded signal, the detection performance of the algorithm degraded as the number of bonded channels increases.
However, the performance degradation is negligible especially as the number of bonded channel increases, and the detection performance is still reliable even though the performance degrades because it is showing better results for negative SNR values. Performance verification is done by comparing detection probability for different number of bonded channels, because there is no conventional method to detect channel bonded signal. Identification of channel bonding information is possible with only one channel sensing by skipping the sensing process for the rest of channels.

\section{References}

[1] Haykin S., "Cognitive radio: brain-empowered wireless communications", IEEE 1. Sel. Areas Commun, vol. 23, no. 2, pp. 201-220, Feb. 2005.

[2] Xiao Ma, "Spectrum Sensing based on Sequential Testing", in Electrical and Electronics Engineering at the university of Canterbury, New Zealand, Dec.2009.

[3] Sunmin Lim et al., "Efficient multi-channel signal detection algorithms for cognitive radio systems in TV whitespace", in Proc. International Conference on World of Wireless, Mobile and Multimedia Networks (WoWMoM), Jun. 2012.

[4] Francisco Paisana, Nicola Marchetti, and Luiz A. DaSilva, "Radar, TV and Cellular Bands: Which SpectrumAccess Techniques for Which Bands?", IEEE Communications Surveys \& Tutorials, vol. 16, NO. 3, pp1193,Third Quarter, 2014

[5] IEEE draft standard for information technology telecommunications and information exchange betweensystems - local and metropolitan area networks specific requirements part I I: wireless LAN medium accesscontrol (MAC) and physical layer (PHY) specifications - Amendment 5: TV white spaces operation, IEEE DraftP802.1laf/D3.0, Oct. 2013.

[6] Gaurang Naik, Sudesh Singhal, Animesh Kumar and Abhay Karandikar, "Quantitative Assessment of TV White Space in India", in Proc. Twentieth National Conference on Communications (NCC), Mar.2014.

[7] "National Frequency Allocation Plan 2011, WPC, Department of Telecom., Govt. of India", http://www.dot.gov.in/as/Draft\%20NFAP-2011.pdf

[8] Sunmin Lim et al., "Novel CR signal detection algorithms for coexistence in TV white space", in Proc. International Conference on ICT Convergence, Sep. 2011.

[9] F. Sheikhy and B. Bing, "Cognitive spectrum sensing and detection using polyphase OFT filter banks", in Proc. Consumer Communications and Networking Conference, Jan. 2008.

[10] Yong Soo Cho, Jaekwon Kim, Won Young Yang, Chung G. Kang, "MIMO-OFDM Wireless Communications with Matlab", John Wiley \& Sons (Asia) Pvt Ltd., 2010.

[11] Hou-Shin Chen et aI., "Spectrum sensing for OFDM systems employing pilot tones", IEEE Tran. Wireless Commun., vol. 8, pp. 5862-5870, Dec. 2009.

[12] Cong Wang et aI., "Multi-window spectrum sensing of unsynchronized OFDM signal at very low SNR", in 
Proc. IEEE Global Telecommunications Conference, Dec. 2009.

[13] X. Wang et aI., "Identification of PCP-OFDM signals at very low SNR for spectrum efficient communications", in Proc. IEEE 69th Vehicular Technology Conference, Apr. 2009.

[14] F. Socheleau et aI., "OFDM system identification for cognitive radio based on pilot-induced cyclostationarity", in Proc. IEEE Wireless Communications and Networking Conference, Apr. 2009.

[15] Mourad Barkat, "Signal Detection and Estimation", Artech House, Inc, Second Edition.

[16] Vatsal Sharan, Prenit Wankhede, "Spectrum Sensing for Cognitive Radio", in Proc. National Conference on Communications (NCC), Dec. 2012. 